\title{
Direct force measurements on DNA in a solid-state nanopore
}

\author{
ULRICH F. KEYSER, BERNARD N. KOELEMAN, STIJN VAN DORP, DIEGO KRAPF, RALPH M. M. SMEETS, \\ SERGE G. LEMAY, NYNKE H. DEKKER* AND CEES DEKKER*
}

Kavli Institute of Nanoscience, Delft University of Technology, 2628 CJ Delft, The Netherlands

*e-mail: N.H.Dekker@tudelft.nl; C.Dekker@tudelft.nl

1 mong the variety of roles for nanopores in biology, an important one is enabling polymer transport, for example in gene transfer between bacteria ${ }^{1}$ and transport of RNA through the nuclear membrane ${ }^{2}$. Recently, this has inspired the use of protein ${ }^{3-5}$ and solid-state $e^{6-10}$ nanopores as single-molecule sensors for the detection and structural analysis of DNA and RNA by voltage-driven translocation. The magnitude of the force involved is of fundamental importance in understanding and exploiting this translocation mechanism, yet so far it has remained unknown. Here, we demonstrate the first measurements of the force on a single DNA molecule in a solid-state nanopore by combining optical tweezers ${ }^{11}$ with ionic-current detection. The opposing force exerted by the optical tweezers can be used to slow down and even arrest the translocation of the DNA molecules. We obtain a value of $0.24 \pm 0.02 \mathrm{pN} \mathrm{mV}^{-1}$ for the force on a single DNA molecule, independent of salt concentration from 0.02 to $1 \mathrm{M} \mathrm{KCl}$. This force corresponds to an effective charge of $0.50 \pm 0.05$ electrons per base pair equivalent to a $75 \%$ reduction of the bare DNA charge.

It is possible to manipulate DNA molecules using electric fields because DNA is negatively charged in solution. Confining an electrical field to a nanopore enables the study of voltagedriven DNA translocation where the force is only applied to the few monomers that are inserted in the nanopore. We can calculate the electrical force $F_{\mathrm{el}}$ on the DNA in the nanopore as $F_{\mathrm{el}}=$ $\int\left(q_{\text {eff }}(z) / a\right) E(z) \mathrm{d} z$, where $q_{\text {eff }}$ is the effective charge of a DNA base pair, $E(z)$ is the position-dependent electrical field in our system, $a$ is the distance between two base pairs, and the integral is taken along the DNA contour. Assuming that $q_{\text {eff }}$ is identical for every base pair leads to $F_{\text {el }}=\left(q_{\text {eff }} / a\right) \int E(z) \mathrm{d} z=q_{\text {eff }} \Delta V / a$, with $\Delta V$ the applied potential across the nanopore. The simplicity of this formula stems from the translational invariance of our system, in which the contour length of the DNA exceeds the length of the nanopore. The introduction of an additional base pair into the nanopore will simply expel an identical base pair from the other side. It follows that the exact geometrical shape of the nanopore or the exact form of $E(z)$ plays no role in the determination of the effective charge $q_{\text {eff }}$ (ref. 12). Published values for $q_{\text {eff }}$ vary widely.

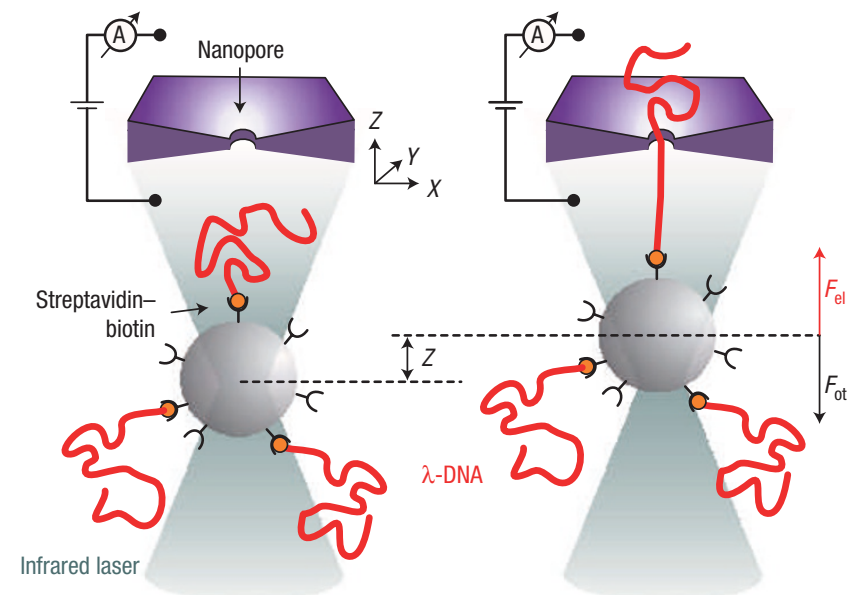

Figure 1 Experimental configuration. A tightly focused laser beam is used to trap a DNA-coated bead near a solid-state nanopore immersed in a saline solution (left image). Application of a voltage drives an ionic current through the nanopore and the negatively charged DNA into the nanopore. When a DNA molecule enters the nanopore (right image), an electrical force $F_{\text {el }}$ is exerted on the bead which is pulled out of the trap centre over a distance $\Delta Z$ until the optical force $F_{\text {ot }}$ and the electrical force are balanced, $F_{\mathrm{el}}=F_{\mathrm{ot}}$.

The upper limit for the force is set by the bare DNA charge of $2 \mathrm{e}^{-} / \mathrm{bp}$, which yields $F_{\mathrm{el}} \sim 100 \mathrm{pN}$ with $a=0.34 \mathrm{~nm}$ and a voltage of $100 \mathrm{mV}$.

We use optical tweezers ${ }^{11,13}$ both as a tool to controllably insert a single DNA molecule into a nanopore and as a probe of $F_{\mathrm{el}}$. In Fig. 1, we show the experimental configuration. A polystyrene bead coated with long DNA molecules $(\lambda$-DNA, $48.5 \mathrm{~kb})$ is trapped using an infrared laser, allowing control of its position in close proximity to the nanopore. Optical traps are characterized by a trap stiffness $k_{\text {trap }}$ that relates the distance $\Delta Z$ of the bead from the trap centre to the restoring optical force $F_{\mathrm{ot}}=-k_{\text {trap }} \Delta Z$. To measure $F_{\mathrm{ot}}$, we 

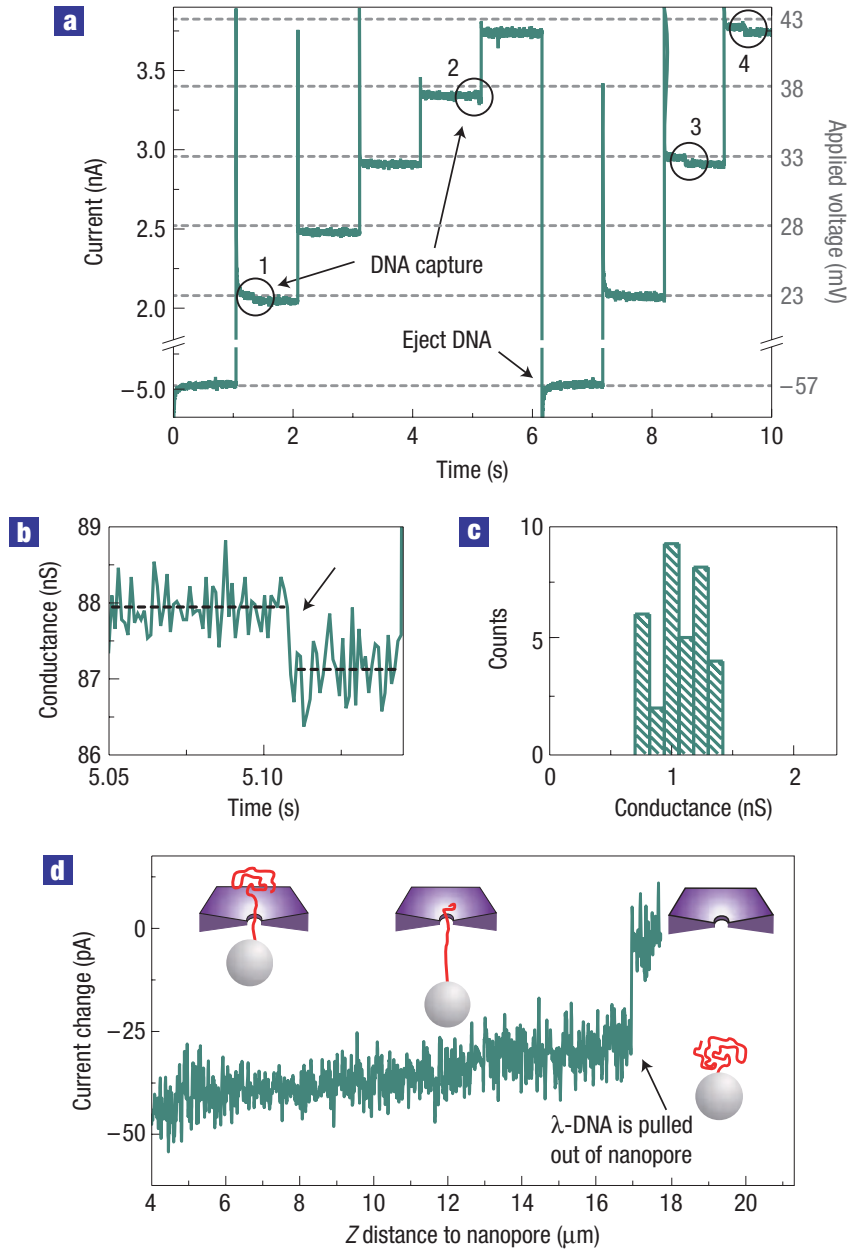

Figure 2 DNA capture events. a, lonic current through a nanopore as a function of time for different constant voltages (indicated on the right-hand side). The circles indicate four events for which the current is step-wise decreased by a DNA molecule entering the nanopore. At event 1 , a first molecule enters the nanopore, and at event 2 a second one enters. After $\sim 6 s$ the voltage is reversed and the current returns to the original open-pore value. Subsequently, the measurements are repeated with two DNA molecules entering the nanopore at events 3 and 4 . The dashed lines show the current values without any DNA in the nanopore. $\mathbf{b}$, A typical conductance step observed when a DNA molecule enters the nanopore. This conductance step corresponds to a zoom of event 2 from a. c, Histogram of 34 conductance steps showing a single peak observed in a single experimental run with the same nanopore. This indicates that one DNA molecule enters at a time. $\mathbf{d}$, Change in current as a function of distance between trap centre and nanopore for a voltage of $30 \mathrm{mV}$. The DNA is pulled out of the nanopore at a speed of $30 \mathrm{~nm} \mathrm{~s}^{-1}$. When the distance exceeds the contour length of the DNA, the DNA is pulled out of the nanopore and the current increases in a step-wise fashion. For this trace we subtracted the current trace when moving away from (with DNA in the nanopore) and towards the nanopore (without DNA in the nanopore).

built optical tweezers that use reflected light from the bead for the position detection ${ }^{14}$. The advantage of such a configuration is that only one high-numerical-aperture objective is required instead of two, which gives freedom to mount a specially designed flow cell incorporating current detection.

An applied voltage over the nanopore drives a DNA molecule into the nanopore as in a simple translocation experiment $t^{7,8,10}$. Insertion of a DNA molecule into the nanopore is detected by two independent measurements: (1) a change in the ionic current due to the partial blockage of the nanopore by the DNA molecule, and (2) a change in the bead position in the optical trap until the optical force $F_{\text {ot }}$ pulling the bead back to the centre of the optical trap is equal and opposite to $F_{\mathrm{el}}$. Thus, the optical trap provides the unique possibility to directly and accurately measure $F_{\mathrm{el}}$ and arrest the translocation process completely. If needed, we can subsequently set the translocation speed to any desired value by moving the optical trap at a given speed towards the nanopore. Alternatively, we can reverse the translocation direction and pull the DNA out of the nanopore against the electrical force.

Figure $2 \mathrm{a}$ shows a typical current measurement when the distance separating the DNA-coated bead from the nanopore is smaller than twice the radius of gyration of the DNA $\left(2 R_{\mathrm{g}} \sim\right.$ $1.2 \mu \mathrm{m})$. At a fixed voltage, we observe that the ionic current changes abruptly when the DNA is captured in the nanopore, as indicated by the circles in Fig. 2a. The typical event shown in Fig. $2 \mathrm{~b}$ emphasizes the clear step-wise decrease in conductance. As expected for this salt concentration of $1 \mathrm{M} \mathrm{KCl}$, the conductance through the nanopore decreases when a DNA molecule enters ${ }^{10}$. We verify that DNA truly is inserted into the nanopore by reversing the polarity of the voltage. As expected, the DNA is driven out of the pore and the original current value is once again recovered (the dashed lines in Fig. 2a indicate the original current values).

The step-wise decrease in conductance due to the capture of a DNA molecule in the nanopore is quantized, as summarized in a histogram in Fig. $2 \mathrm{c}$ showing a single peak at $1.1 \mathrm{nS}$. All 34 data points were collected during one experiment with the same nanopore at different voltages, and thus different currents. The step size and the width of the distribution are in very good agreement with values that we measure for translocation events of untethered DNA molecules. This confirms that only one DNA molecule enters the nanopore at a time, which directly implies that we can determine the number of DNA molecules in the nanopore by counting the number of conductance steps in a trace such as that shown in Fig. 2a. We reproduced the controlled capture of DNA in the nanopore in more than 10 experiments with different nanopores, with diameters ranging from 6 to $15 \mathrm{~nm}$.

Increasing the distance between the nanopore and the DNAcoated bead allows us to tune the probability of DNA entering the nanopore. Experimentally we observe no frequent capture events when the distance exceeds the radius of gyration of the $\lambda$-DNA fourfold. Tuning both the distance and the voltage allows us to capture only a single DNA molecule in the nanopore in a wellcontrolled manner. When captured, the DNA molecule is pulled taut between the nanopore and the bead. This is demonstrated by monitoring the ionic current while increasing the distance between the optical trap centre and the nanopore until it exceeds the DNA contour length (Fig. 2d). Here, the DNA is controllably pulled at a speed of only $30 \mathrm{~nm} \mathrm{~s}^{-1}$, this is more than five orders of magnitude slower than the usual translocation of $\sim 8 \mathrm{~mm} \mathrm{~s}^{-1}$ for free DNA calculated from the histogram in Fig. 3c. At a distance of $16.9 \mu \mathrm{m}$, the DNA is pulled out of the nanopore, indicated by the sharp increase in current. This value is in good agreement with the sum of the contour length of $\lambda$-DNA of $16.5 \mu \mathrm{m}$ and the $1.1-\mu \mathrm{m}$ radius of our beads. The additional slow decrease of the current is due to the diminishing influence of the heat generated in the laser focus, as described elsewhere ${ }^{15}$.

Figure 3 shows the force-induced response of the bead to the electrical force acting on the DNA in the nanopore. First in Fig. 3a, we show the nanopore current and the position signal of a bead in the optical trap for a typical capture event. It can be seen that the current and the bead position change nearly simultaneously. However, the change in bead position $\Delta Z$ is always slightly delayed with respect to the current response. This delay time $\Delta t$ (Fig. 3a) 

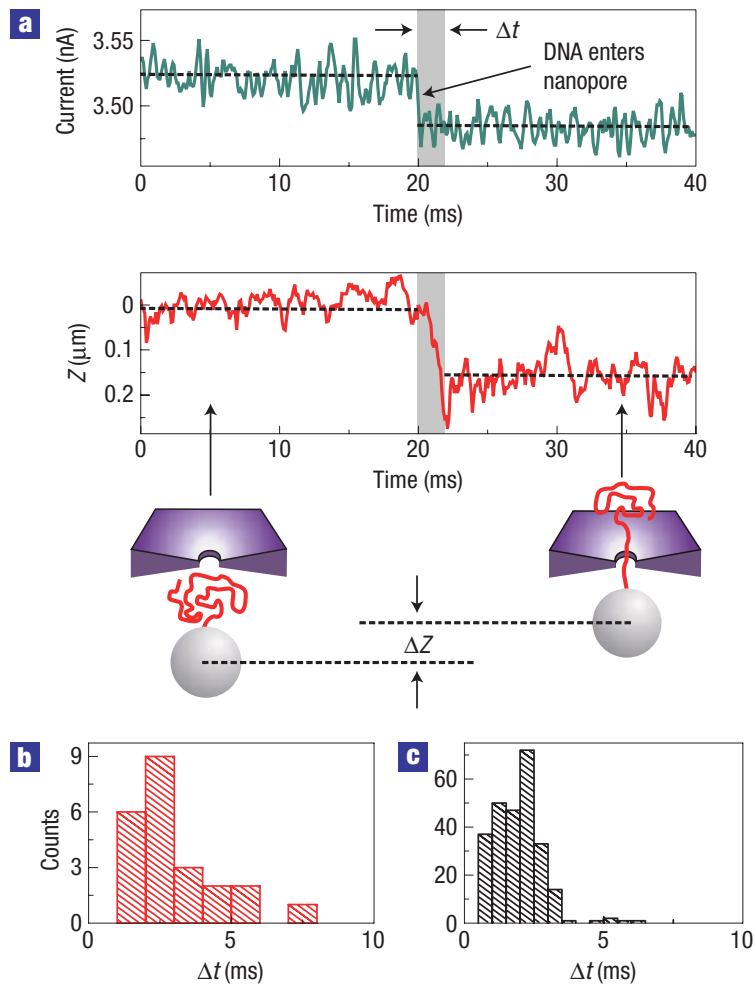
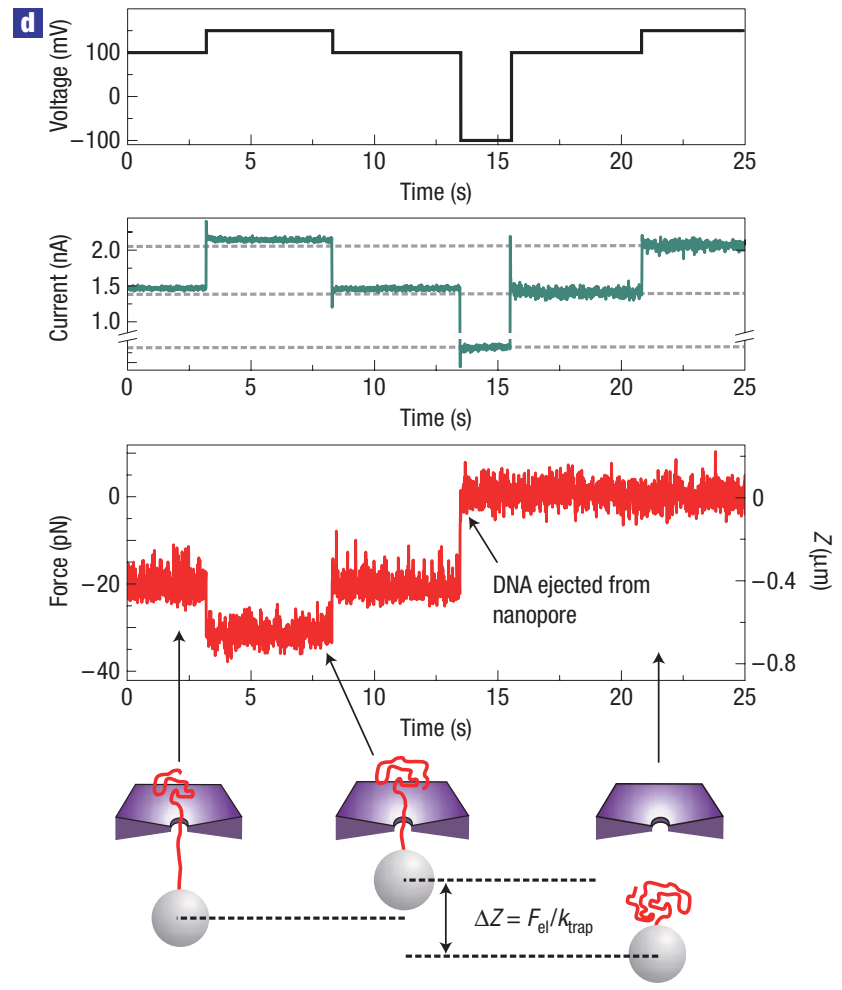

Figure 3 Simultaneous force and ionic-current response on DNA in the nanopore. a, Current and position as a function of time for one capture event at $1 \mathrm{M} \mathrm{KCl}$ and a voltage of $33 \mathrm{mV}$ at a bandwidth of $10 \mathrm{kHz}$. When the DNA enters the nanopore, the ionic current drops. After a delay time $\Delta t$ required to pull the DNA taut, the bead responds to the electrical force pulling on the DNA. $\mathbf{b}$, Histogram for the delay time $\Delta t$ that it takes to pull the DNA taut. The capture voltage varied between 30 and $80 \mathrm{mV}$. c, Histogram of the translocation time for untethered $\lambda$-DNA through the nanopore at $1 \mathrm{M} \mathrm{KCl}$ and $50 \mathrm{mV}$, showing similarity to $\Delta t$ in $\mathbf{b}$. $\mathbf{d}$, Current (green) and force (red) as a

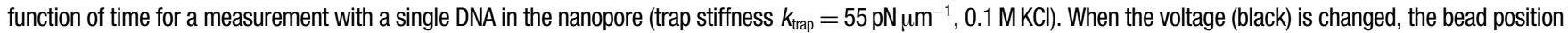
changes until $F_{\mathrm{el}}=F_{\mathrm{ot}}$. At $t=13 \mathrm{~s}$ we drive the DNA out of the nanopore by reversing the voltage and subsequently, as expected, the bead no longer responds to further voltage changes while the current returns to the open-pore current (dashed lines). Note that at this tenfold lower salt concentration, the presence of DNA actually leads to an increase rather than a decrease in the ionic current through the nanopore ${ }^{29}$.

reflects the time difference between the current blockade by the DNA in the nanopore and the bead reaching its new equilibrium position in the optical trap where $F_{\mathrm{el}}=F_{\mathrm{ot}}$. This result is easily understood by realizing that the current blockade will be complete as soon as the DNA has entered the nanopore but that the bead only responds to the force exerted on the DNA when the DNA is pulled taut between the nanopore and the bead. As the distance between the bead and the nanopore is $2 \mu \mathrm{m}$ in this experiment, first $14 \mu \mathrm{m}$ of DNA has to pass through the nanopore, resulting in the delay time $\Delta t$. Figure $3 \mathrm{~b}$ shows a histogram for $\Delta t$ recorded for numerous (re)captures of a single DNA molecule, yielding a mean value of $2.5 \pm 1.5 \mathrm{~ms}$. This agrees very well with the translocation-time histogram measured with untethered $\lambda$-DNA $(16.5 \mu \mathrm{m})$ shown in Fig. $3 c$, which yields a mean translocation time of $1.9 \pm 0.8 \mathrm{~ms}$.

Having established the controllable presence of a single DNA molecule in the nanopore, we expect to be able to quantitatively measure the bead position as a function of the applied voltage, leading to a determination of the electrical force $F_{\mathrm{el}}$ acting on the DNA. Figure $3 \mathrm{~d}$ shows measurements with a DNA molecule already in the nanopore at a salt concentration of $0.1 \mathrm{M} \mathrm{KCl}$. If a DNA molecule is present in the nanopore, the bead position $\Delta Z$ should follow the voltage changes because $F_{\text {ot }}=k_{\text {trap }} \Delta Z=F_{\text {el }}=$ $q_{\text {eff }} \Delta V / a$. Our experimental verification is shown in Fig. 3d, which demonstrates that the bead position indeed closely reflects the changes in applied voltage. Subsequently at $t=13 \mathrm{~s}$, we reverse the voltage and drive the DNA out of the nanopore. Figure $3 \mathrm{~d}$ confirms that voltage changes have no effect on bead position during the remainder of the measurement when no DNA is in the nanopore.

From such experiments, we directly deduce the force acting on the DNA. A typical result is shown in Fig. 4. We measured the force on the DNA as a function of applied voltage at three different separations between the nanopore and the trap centre at $0.1 \mathrm{M} \mathrm{KCl}$. The data overlap nicely, showing that the force depends on neither the distance between the nanopore and the bead, nor the length of DNA on either side of the nanopore, as expected. Fitting the data with a linear function yields a slope of $0.25 \pm 0.03 \mathrm{pN} \mathrm{mV}^{-1}$ in $0.1 \mathrm{M} \mathrm{KCl}$. The error stems from the uncertainty of the calibration of the optical trap and is in line with values published in the literature. We reproduced this value in experiments with nanopores of different sizes $(6-11 \mathrm{~nm})$ and carried out measurements over a range of salt concentrations from $20 \mathrm{mM} \mathrm{KCl}$ to $1 \mathrm{M} \mathrm{KCl}$. The inset of Fig. 4 shows the slopes for these measurements as a function of $\mathrm{KCl}$ concentration (see Supplementary Information, Fig. S1 for full traces). The average slope of $0.24 \mathrm{pN} \mathrm{mV}^{-1}$ is shown as a horizontal line. Despite a variation of ion concentration by a factor of 50, the forces acting on the molecule seem unchanged. A control measurement in $0.2 \mathrm{M} \mathrm{NaCl}$ (see Supplementary Information, Fig. S1) yielded the same value for the force within the experimental error.

Our direct measurements provide the first determination of the force acting on a biopolymer during translocation in a nanopore. Estimates with values from the literature ranged from 0.05 (ref. 16) 


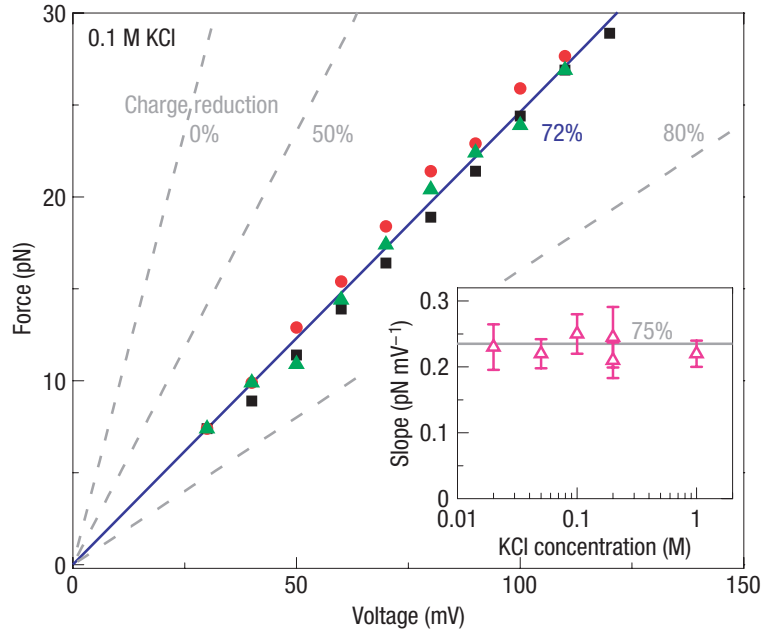

Figure 4 Force measurements on a single DNA molecule in a nanopore. Force as a function of voltage at $0.1 \mathrm{M} \mathrm{KCl}$ recorded for one molecule at three different distances from the nanopore (filled squares $2.1 \mu \mathrm{m}$, filled circles $2.4 \mu \mathrm{m}$, filled triangles $2.9 \mu \mathrm{m})$. The dashed lines are forces calculated for reduction of the DNA charge of $0 \%$ (bare DNA), $50 \%$, and $80 \%$. The solid blue line is a fit through the data, which yields a slope of $0.25 \pm 0.03 \mathrm{pN} \mathrm{mV}^{-1}$. The inset shows the slopes as a function of salt concentration obtained from measurements in $0.02-1.0 \mathrm{M} \mathrm{KCl}$. The line denotes the average value of $0.24 \mathrm{pN} \mathrm{mV}^{-1}$. The error bars result from errors in the calibration of the optical trap.

to 0.5 (ref. 17) $\mathrm{pN} \mathrm{mV}^{-1}$, on the basis of the electrophoretic mobility of DNA. The deduction of the absolute forces from our results will guide further modelling of translocation experiments, and opens up the route towards quantitative force spectroscopy with a solid-state nanopore, for example, for quantitative studies of DNA-protein unbinding.

In our experiments, the nanopore diameter $(6-11 \mathrm{~nm})$ is significantly larger than the diameter of double-stranded DNA of $2.2 \mathrm{~nm}$. This minimizes the interaction (friction, sticking) between the DNA and the nanopore walls, prevents Debye overlap between the screening clouds of DNA and the surface, and avoids wetting problems encountered with very small $(<3 \mathrm{~nm})$ nanopores ${ }^{18}$. One possible complication is the negatively charged surface of the nanopore. It follows that an additional electro-osmotic flow is present in the nanopore, exerting a finite drag on the DNA molecule. However, the correction introduced by this drag force is only of the order of a few per cent of the total force and can safely be ignored ${ }^{12,18}$.

From the force values we can directly deduce the effective charge of the DNA in our nanopores. Using $F_{\mathrm{el}}=q_{\mathrm{eff}} \Delta V / a$, we calculate from the average slope of $0.24 \mathrm{pN} \mathrm{mV}^{-1}$ that the effective charge of DNA is constant $(0.02 \mathrm{M}-1.0 \mathrm{M} \mathrm{KCl})$ and equals $q_{\text {eff }}=0.50 \pm$ 0.05 elementary charges per base pair, corresponding to a charge reduction of $75 \%$.

Until now the value of the effective charge was extracted from indirect experimental results from gel electrophoresis. Extensive modelling was required to convert the measured DNA mobility into effective charge $e^{16,17,19-22}$. Values published in the literature vary widely over a range from $0.12 \mathrm{e}^{-} / \mathrm{bp}$ (refs 16 , 21 ) to $1.0 \mathrm{e}^{-} / \mathrm{bp}$ (ref. 17). In contrast, our experiments yield $q_{\text {eff }}=0.50 \pm 0.05 \mathrm{e}^{-} / \mathrm{bp}$, which is constant for the range of ion concentrations probed here $(0.02-1 \mathrm{M} \mathrm{KCl})$. Our value is close to the value of $0.48 \mathrm{e}^{-} / \mathrm{bp}$ for counterion condensation as predicted by Manning ${ }^{23}$, which is remarkable given the complex interplay between hydrodynamics and electrical charges in the screening layer of the DNA. Our data may provide a basis for developing a microscopic theory of the dynamics of ions on DNA.

In conclusion, we have succeeded in measuring for the first time the electrical force acting on a single DNA molecule in a nanopore, and from these data we determined the effective charge of DNA in salt solutions. In addition, the combination of optical tweezers and ionic-current measurements introduces a new and versatile singlemolecule technique that enables new biophysical experiments such as the sequential unfolding of RNA hairpins and nanometre-precise spatial detection of proteins bound to $\mathrm{DNA}^{24}$. As we are able to hold and control the translocation speed of DNA in a nanopore, our new technique may even open a way to sequence DNA with solidstate nanopores, because optical tweezers with single base-pair resolution $^{11,13}$ and DNA translocation through $2 \mathrm{~nm}$ nanopore ${ }^{25}$ have been demonstrated.

\section{METHODS}

\section{NANOPORE FABRICATION}

The nanopores (with diameters between 6 and $15 \mathrm{~nm}$ ) are fabricated in $20 \mathrm{~nm}$ thin low-stress silicon nitride $(\mathrm{SiN})$ membranes ${ }^{9}$. Fabrication starts with the deposition of a sandwich layer on silicon using low-pressure chemical vapour deposition, yielding a $20 \mathrm{~nm}$ thin SiN layer, followed by $200 \mathrm{~nm}$ of tetra-ethyl-ortho-silicate $\mathrm{SiO}_{2}$, and finally $500 \mathrm{~nm}$ of SiN. A freestanding membrane is obtained by etching the silicon in $\mathrm{KOH}$. In the centre, this membrane is thinned over a circular region of $5 \mu \mathrm{m}$ diameter by removing the top two layers, using reactive ion etching and hydrofluoric acid. Next, $20 \mathrm{~nm}$ thin $\mathrm{SiO}_{2}$ is sputtered onto the frontsides and backsides of the membrane to make the surface hydrophilic. Nanopores are drilled in a transmission electron microscope by tightly focusing the electron beam on the $60 \mathrm{~nm}$ thin $\mathrm{SiO}_{2} / \mathrm{SiN} / \mathrm{SiO}_{2}$ membrane. The focused electron beam leads to the formation of a small hole by sputtering atoms from the membrane.

\section{IONIC-CURRENT MEASUREMENT AND FLOW CELL}

The nanopores are mounted into our custom-made inverted microscope using a microfluidic polydimethylsiloxane flow cell designed with an optical window. We facilitate wetting of the nanopores by a $30 \mathrm{~s}$ cleaning step in an oxygen plasma before use. Both sides of the nanopores are flushed with $\mathrm{KCl}$ solution together with $10 \mathrm{mM}$ Tris-HCl, $1 \mathrm{mM}$ EDTA (TE) buffer at $\mathrm{pH}=8.0$. Detection of the ionic current is achieved with platinum wires that are immersed in a separate compartment containing $1 \mathrm{M} \mathrm{KCl}$ together with $1 \mathrm{mM}$ potassium-ferri-cyanide and potassium-ferro-cyanide and connected by agarose-gel salt bridges to the flow cell. The signals from the current amplifier (Axopatch 200B) are filtered at $1 \mathrm{kHz}$ and recorded using a LabView-program interface. The experiments are carried out in a custom-built microfluidic sample cell that consists of a $180 \mu \mathrm{m}$ thin polydimethylsiloxane layer on a microscope slide (thickness $180-210 \mu \mathrm{m}$ ) with two fluid inlets and one outlet used to flush in buffer and bead solutions, respectively. The setup will be described in detail elsewhere.

\section{DNABEAD CONSTRUCT}

$\lambda$-DNA ( $48.5 \mathrm{~kb})$ with a single biotin attached to one end is attached to streptavidin-coated polystyrene beads (diameter $2.2 \mu \mathrm{m}$ or $1.94 \mu \mathrm{m}$ ). After washing the beads in $0.1 \mathrm{M} \mathrm{KCl}, 10 \mathrm{mM}$ Tris-HCl $1 \mathrm{mM}$ EDTA (TE) buffer at $\mathrm{pH}=8.0$, DNA is mixed with the beads at a ratio of 300-100:1 in $1 \mathrm{M} \mathrm{KCl}$, $10 \mathrm{mM}$ TE-buffered solution $(\mathrm{pH}=8.0)$. After another washing step, the beads are suspended in the measurement buffer containing $\mathrm{KCl}$ solution with TE buffer $(\mathrm{pH}=8.0)$ and $0.1 \%$ Tween 20 (Sigma Aldrich).

\section{NANOPORE CHARACTERIZATION AND TRAP CALIBRATION}

Before the actual experiments, each nanopore is characterized by current-voltage measurements to check for linear conductance and stability. We check for the absence of voltage-dependent $1 / f$ noise, which is often observed for $\mathrm{SiN}$ nanopores ${ }^{26}$. If the nanopore meets the requirements for low noise and high stability, we record a calibration file very similar to magnetic tweezers measurements by recording the height-dependent diffraction pattern $I(z)$ of the circular membrane ${ }^{27}$. This gives an independent measure for the 
absolute distance between the trap centre and the nanopore during the experiments with an accuracy of roughly $50 \mathrm{~nm}$. For the high-resolution position measurement we use a red laser that is coupled to the objective (collinear to the infrared trapping laser) and focused on the bead. The reflected intensity from the bead is modulated by interference from light reflected from the solid-state membrane. This signal is used to measure the $z$ position of the bead relative to the trap centre with an accuracy better than $5 \mathrm{~nm}$. Our approach is similar to a technique recently described in the literature ${ }^{28} . F_{\text {ot }}$ is linearly proportional to $\Delta Z$ over a range of up to $\pm 700 \mathrm{~nm}$.

Beads with DNA are flushed into the cell, trapped with the optical tweezers setup, and brought close to the nanopore. When initially trapping the DNA-coated bead, the voltage across the nanopore is applied such that no DNA will enter the nanopore. After trapping one bead, $5 \mu \mathrm{l}$ of buffer without beads is flushed through the cell, which has a volume of $1 \mu$ l. For each bead, the trapping potential is characterized by measuring the power spectrum and extracting the corner frequency of the trap. The trap stiffness and corresponding drag coefficient of the bead are checked by measuring the Stokes drag on the bead by moving the bead through the liquid at speeds from 10 to $100 \mu \mathrm{m} \mathrm{s}^{-1}$ with a closed-loop piezo stage (Physik Instrumente). The typical

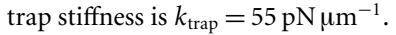

Received 23 September 2005; accepted 2 June 2006; published 1 July 2006.

\section{References}

1. Ochman, H., Lawrence, J. G. \& Groisman, E. A. Lateral gene transfer and the nature of bacterial innovation. Nature 405, 299-304 (2000).

2. Nakielny, S. \& Dreyfuss, G. Transport of proteins and RNAs in and out of the nucleus. Cell 99, 677-690 (1999)

3. Kasianowicz, J. J., Brandin, E., Branton, D. \& Deamer, D. W. Characterization of individual polynucleotide molecules using a membrane channel. Proc. Natl Acad. Sci. USA 93, 13770-13773 (1996)

4. Akeson, M., Branton, D., Kasianowicz, J. J., Brandin, E. \& Deamer, D. W. Microsecond time-scale discrimination among polycytidylic acid, polyadenylic acid, and polyuridylic acid as homopolymers or as segments within single RNA molecules. Biophys. J. 77, 3227-3233 (1999).

5. Meller, A., Nivon, L., Brandin, E., Golovchenko, J. \& Branton, D. Rapid nanopore discrimination between single polynucleotide molecules. Proc. Natl Acad. Sci. USA 97, 1079-1084 (2000).

6. Li, J. et al. Ion-beam sculpting at nanometre length scales. Nature 412, 166-169 (2001).

7. Li, J., Gershow, M., Stein, D., Brandin, E. \& Golovchenko, J. A. DNA molecules and configurations in a solid-state nanopore microscope. Nature Mater. 2, 611-615 (2003).

8. Heng, J. et al. Sizing DNA using a nanometer-diameter pore. Biophys. J. 87, 2905-2911 (2004).

9. Storm, A J Chen J H. Ling X. S. Zandbergen, H. W. \& Dekker, C. Fabrication of solid-state nanopores with single-nanometre precision. Nature Mater. 2, 537-540 (2003).

10. Storm, A. et al. Fast DNA translocation through a solid-state nanopore. Nano Lett. 5, 1193-1197 (2005).
11. Bustamante, C., Bryant, Z. \& Smith, S. Ten years of tension: single-molecule DNA mechanics. Nature 421, 423-427 (2003).

12. Lubensky, D. \& Nelson, D. Driven polymer translocation through a narrow pore. Biophys. J. 77, 1824-1838 (1999).

13. Neuman, K. \& Block, S. Optical trapping. Rev. Sci. Instrum. 75, 2787-2809 (2004).

14. Shivashankar, G., Stolovitzky, G. \& Libchaber, A. Backscattering from a tethered bead as a probe of DNA flexibility. Appl. Phys. Lett. 73, 291-293 (1998).

15. Keyser, U. F. et al. Nanopore tomography of a laser focus. Nano Lett. 5, 2253-2256 (2005).

16. Smith, S. \& Bendich, A. Electrophoteric charge-density and persistence length of DNA as measured by fluorescence microscopy. Biopolymers 29, 1167-1173 (1990).

17. Schellman, J. A. \& Stigter, D. Electrical double layer, zeta potential, and electrophoretic charge of double-stranded DNA. Biopolymers 16, 1415-1434 (1977).

18. Ho, C. et al. Electrolytic transport through a synthetic nanometer-diameter pore. Proc. Natl Acad. Sci. USA 102, 10445-10450 (2005).

19. Laue, T. M. et al. Insights from a new analytical electrophoresis apparatus. J. Pharm. Sci. 85, 1331-1335 (1996).

20. Long, D., Viovy, J. L. \& Ajdari, A. Simultaneous action of electric fields and nonelectric forces on polyelectrolyte: Motion and deformation. Phys. Rev. Lett. 76, 3858-3861 (1996).

21. Gurrieri, S., Smith, S. B. \& Bustamante, C. Trapping of megabase-sized DNA molecules during agarose gel electrophoresis. Proc. Natl Acad. Sci. USA 96, 453-458 (1999).

22. Stellwagen, E. \& Stellwagen, N. C. Probing the electrostatic shielding of DNA with capillary electrophoresis. Biophys. J. 84, 1855-1866 (2003).

23. Manning, G. S. The molecular theory of polyelectrolyte solutions with applications to the electrostatic properties of polynucleotides. Q. Rev. Biophys. 11, 179-246 (1978).

24. Koch, S. J. \& Wang, M. D. Dynamic force spectroscopy of protein-DNA interactions by unzipping DNA. Phys. Rev. Lett. 91, (2003).

25. Heng, J. B. et al. Stretching DNA using the electric field in a synthetic nanopore. Nano Lett. 5, 1883-1888 (2005).

26. Chen, P. et al. Atomic layer deposition to fine-tune the surface properties and diameters of fabricated nanopores. Nano Lett. 4, 1333-1337 (2004).

27. Strick, T. R., Allemand, J. F., Bensimon, D., Bensimon, A. \& Croquette, V. The elasticity of a single supercoiled DNA molecule. Science 271, 1835-1837 (1996).

28. Neuman, K., Abbondanzieri, E. \& Block, S. Measurement of the effective focal shift in an optical trap. Opt. Lett. 30, 1318-1320 (2005).

29. Smeets, R. M. M. et al. Salt dependence of ion transport and DNA translocation through solid-state nanopores. Nano Lett. 6, 89-95 (2006).

\section{Acknowledgements}

We thank M.-Y. Wu and H. Zandbergen for help in fabrication of the nanopores, B. Quinn, D. Stein, D. Lubensky, and R. Seidel for useful discussions, P. Veenhuizen and S. Hage for preparing the DNA constructs, and K. Klein for taking the data with the untethered DNA. J. van der Does is acknowledged for help in designing and building the flow cell and mechanical parts of the tweezers setup. We thank NWO and FOM for financial support.

Correspondence and requests for materials should be addressed to C.D

Supplementary Information accompanies this paper on www.nature.com/naturephysics.

\section{Competing financial interests}

The authors declare that they have no competing financial interests.

Reprints and permission information is available online at http://npg.nature.com/reprintsandpermissions/ 